Prävention hat ihren Preis und gerät deshalb insbesondere dort unter Beschuss, wo sie die öffentlichen Kassen belastet. In Frage steht dabei nicht die präventive Vernunft als solche, sondern wer ihr Geltung verschaffen soll. Im Zuge der gegenwärtigen Ökonomisierung des Sozialen verwandelt sich der „Vorsorgestaat” (Ewald, 1993) zum aktivierenden Staat, der seine Bürger und Bürgerinnen aus der fürsorglichen Belagerung in die Freiheit der Selbstsorge entlässt und ihnen zumutet, ihre Lebensrisiken eigenverantwortlich zu managen. Gesundheitspolitische Maßnahmen richten sich kaum mehr unmittelbar auf individuelles oder kollektives Verhalten, sondern verändern stattdessen Anreizstrukturen. Nicht die pastorale Umsicht einer gleichermaßen behütenden wie kontrollierenden Verwaltung, sondern marktförmige Selbstregulation soll die optimale Allokation knapper Ressourcen gewährleisten und Risikominimierung mit Autonomiemaximierung verbinden. Die Individuen werden als Unternehmer ihres eigenen Lebens adressiert, die ihr leibliches und seelisches Wohlbefinden als wertvolles asset verwalten und zu steigern versuchen. Prävention wird zur Anlagestrategie: Wer sich in der Wettbewerbsgesellschaft behaupten will, tut gut daran, rechtzeitig ins eigene $\mathrm{Hu}$ mankapital zu investieren.
Aktuelle Präventionsstrategien setzen im Unterschied zu den traditionellen Mechanismen der Aufklärung und Verbote deshalb verstärkt auf ein Regime freiwilliger Selbstkontrolle. Kompetenz- und Ressourcenorientierung lauten die Schlagworte, und nicht nur in der Suchtprävention hat sich inzwischen die Erkenntnis durchgesetzt, die Stärken zu stärken, sei wirksamer, als Ängste zu schüren oder Verbote auszusprechen. Ohne Drohszenarien kommt indes auch der Appell an die Selbstverantwortung nicht aus: Wer es an Einsicht fehlen lässt und etwa auf Tabak oder Alkohol nicht verzichten will, wer keinen Sport treibt oder regelmäßige Vorsorgeuntersuchungen versäumt, der hat auch die Folgen selbst zu tragen - seien es in Form höherer Versicherungsprämien, sei es in Form einer geringeren Lebenserwartung. Je dichter das Netz präventiver Interventionsmöglichkeiten, desto fahrlässiger handelt, wer sie nicht wahrnimmt. Vorbeugung avanciert zum moralischen Imperativ, dessen Unabweisbarkeit gerade darauf beruht, dass er nicht an höhere Ideale, sondern an das Eigeninteresse appelliert. Weil dieser Imperativ sich auf alle Lebensbereiche erstreckt, ist ihm eine ebenso universelle Schuldzuweisung eingeschrieben. Welche kleinen oder großen Katastrophen die Einzelnen auch ereilen mögen, in letzter
Konsequenz sind sie stets ein Ergebnis ihrer unzureichenden Sorge um sich. ,[D]ie meisten (wenn nicht alle!) Todesfälle [sind] bis zu einem gewissen Grade ,Selbstmorde", heißt es bei Gary S. Becker (1982, 9), Nobelpreisträger für Ökonomie und Mitbegründer der Humankapitaltheorie, ,in dem Sinne, dass man sie hätte hinausschieben können, wenn man mehr Ressourcen in die Lebensverlängerung investiert hätte.” Dieser Logik des ,,selber schuld" entgeht niemand, denn der Ausgang allen präventiven Bemühens steht immer schon fest: In the long run we are all dead. Vorbeugung gewährt allenfalls Aufschub. Vielleicht ist das der Grund für das konstitutiv schlechte Gewissen, dass Präventionisten haben - und anderen machen.

Der korrespondierende Autor erklärt, dass kein Interessenkonflikt vorliegt.

Literatur siehe Literatur zum Schwerpunktthema.

http://journals.elsevier.de/pubhef/literatur

http://dx.doi.org/10.1016/j.phf.2013.09.003

Prof. Dr. Ulrich Bröckling

Albert-Ludwigs-Universität Freiburg

Institut für Soziologie

Rempartstr. 15

79098 Freiburg

ulrich.broeckling@soziologie.uni-

freiburg.de

\title{
Positionen zivilgesellschaftlicher Akteure und von Parteien zu Wettbewerb im Gesundheitswesen
}

\author{
Martin Brünger, Maike Grube und Nina Buttmann
}

Anlässlich der Bundestagswahl 2013 haben sich sowohl Parteien als auch zivilgesellschaftliche Akteure zur Rolle des Wettbewerbs im Gesundheitswesen positioniert. Zentrale Konfliktlinie in der Debatte bildet der System- wettbewerb zwischen Gesetzlicher (GKV) und Privater Krankenversicherung (PKV). Daneben wird über Ausgestaltung und Ausmaß des Wettbewerbs zwischen einzelnen Krankenversicherungen diskutiert. Weitere
Fragen der wettbewerblichen Ausgestaltung des Gesundheitswesens, wie z.B. Wettbewerb zwischen Leistungserbringern oder im Arzneimittelbereich, sowie der Vertragswettbewerb (Kollektiv- versus Selektivverträge) 
spielen in der aktuellen politischen Diskussion entweder eine untergeordnete Rolle oder werden weniger kontrovers gehandelt.

Vehementester Verfechter des dualen Krankenversicherungssystems auf Seiten der Parteien ist die FDP (FDP, 2013). Auch die Unionsparteien plädieren für den Systemwettbewerb von GKV und PKV (CDU, 2013), obgleich es in der CDU durchaus kritische Stimmen gibt (Deutsches Ärzteblatt, 2012). Die bürgerlichen Parteien befürworten daneben die Stärkung des Wettbewerbs innerhalb der GKV, z.B. durch unterschiedlich hohe Zusatzprämien einzelner Krankenkassen und die Möglichkeit von Wahltarifen und Prämienrückerstattungen. Kritisiert wird, dass sich der Wettbewerb innerhalb der PKV auf Neuversicherte beschränkt, da es aufgrund unzureichender Portabilitätsmöglichkeiten von Altersrückstellungen aktuell kaum Wettbewerb um Bestandsversicherte gibt (Bundesärztekammer, 2013; Frankfurter Allgemeine Zeitung, 2013). Neben der PKV selbst (Verband der Privaten Krankenversicherung, 2011) befürworten auch Arbeitgeberverbände den Fortbestand des dualen Systems (Bundesvereinigung der Deutschen Arbeitgeberverbände, 2010). Wettbewerb von GKV und PKV sowie zwischen einzelnen Krankenversicherungen diene der Verbesserung von Qualität und Effizienz der Versorgung.

Interessenvertretungen der Ärzteschaft treten bis auf wenige Ausnahmen (z.B. Verein Demokratischer Ärztinnen und Ärzte, 2012) ebenfalls entschieden für den Wettbewerb der beiden Versicherungssysteme ein. Ein einheitlicher Versicherungsmarkt mit gleichen Zugangsbedingungen für alle Bürgerinnen und Bürger wird klar abgelehnt (Bundesärztekammer, 2013; Hartmannbund, 2013; Kassenzahnärztliche Bundesvereinigung, 2013;
Marburger Bund, 2009). Ein wesentlicher Grund für diese Position stellt die höhere Vergütung ärztlicher Leistungen in der PKV dar (Bundesärztekammer, 2012; Kassenärztliche Bundesvereinigung, 2010). Umfragen unter Ärztinnen und Ärzten ergeben allerdings ein differenzierteres Bild. So befürwortet laut Institut für Demoskopie Allensbach die Mehrheit der Ärztinnen und Ärzte die Einführung eines einheitlichen Versicherungsmarkts in Form einer Bürgerversicherung (MLP AG, 2013).

SPD, Grüne und Die Linke treten für die Abschaffung des dualen Krankenversicherungssystems und die Errichtung einer einheitlichen Wettbewerbsordnung ein (Bündnis 90/Die Grünen, 2013; Die Linke, 2013; HeinrichBöll-Stiftung, 2013; SPD, 2013). Neben der Notwendigkeit einer nachhaltigeren Finanzierungsgrundlage des Gesundheitswesens werden Ungerechtigkeiten im aktuellen System als Argument für ein einheitliches Versicherungssystem angeführt: Ungleiche und restriktive Zugangsbedingungen in GKV und PKV, negative Risikoauslese in der PKV aufgrund fehlenden Kontrahierungszwangs und risikoäquivalenter Prämien sowie unterschiedliche Vergütungssysteme, welche insbesondere im ambulanten Sektor Fehlanreize setzen und die sich nicht am Gesundheitsnutzen für den Patienten orientieren.

Unterstützung erfährt dieses Anliegen u.a. von Gewerkschaften (Deutscher Gewerkschaftsbund, 2011), Sozialund Wohlfahrtsverbänden (Arbeiterwohlfahrt, 2010; Diakonie, 2009; Paritätischer Wohlfahrtsverband, 2011; Sozialverband Deutschlands, 2013; Sozialverband VdK, 2010) sowie Patientenvertretungen (Bundesarbeitsgemeinschaft der PatientInnenstellen, 2003; Verbraucherzentrale Bundesverband, 2010). So mahnt der Deutsche Gewerkschaftsbund die ungleichen 
Bedingungen für GKV und $\mathrm{PKV}$ an und hat mit Unterstützung von Mitgliedsgewerkschaften und weiteren Verbänden eine eigene Konzeptskizze für ein Bürgerversicherungsmodell erarbeitet (Deutscher Gewerkschaftsbund, 2011). Auch der Verbraucherschutzverband fordert einen einheitlichen Wettbewerbsrahmen und eine einheitliche Gebührenordnung, um aktuell bestehende Ungleichbehandlungen zu beenden (Bertelsmann Stiftung, 2013; Verbraucherzentrale Bundesverband, 2010).

Wettbewerb zwischen einzelnen Krankenkassen z.B. mittels Beitragssatz- oder Vertragsautonomie wird von Akteuren befürwortet, wenn dies zu wirksameren und kosteneffizienteren Versorgungsstrukturen führt (z.B. GKV-Spitzenverband, 2013; Sozialverband Deutschlands, 2013; SPD, 2013). Dies setzt eine adäquate Kom- pensation der unterschiedlichen Versichertenstruktur einzelner Krankenversicherungen voraus. Daher wird eine Nachjustierung des morbiditätsorientierten Risikostrukturausgleichs gefordert (z.B. Bundesarbeitsgemeinschaft Selbsthilfe, 2013; Diakonie, 2009).

Die Analyse der Positionen von Parteien und zivilgesellschaftlichen Akteuren zeigt deutlich divergierende Standpunkte hinsichtlich des Systemwettbewerbs. Kursorisch sind diese in Abbildung 1 zusammengefasst. Auf der einen Seite gibt es Befürworter des dualen Systems aus GKV und $\mathrm{PKV}$ in den bürgerlichen Parteien, in Arbeitgeberverbänden und ärztlichen Interessensvertretungen, die eine hochwertige Versorgung durch die bestehenden Strukturen gewährleistet sehen. Auf der anderen Seite stehen die Parteien links der Mitte, Gewerk- schaften, Sozial- und Wohlfahrtsverbände sowie Patientenvertretungen, die ein einheitliches Versicherungssystem schaffen wollen, um größere Gerechtigkeit unter Versicherten sowohl hinsichtlich der Bemessung der Beiträge als auch einer bedarfsgerechten Versorgung sicherzustellen.

Der korrespondierende Autor erklärt, dass kein Interessenkonflikt vorliegt.

Literatur siehe Literatur zum Schwerpunktthema.

http://journals.elsevier.de/pubhef/literatur

http://dx.doi.org/10.1016/j.phf.2013.09.005

Martin Brünger, MPH

Charité - Universitätsmedizin Berlin

Centrum für Human- und

Gesundheitswissenschaften

Charitéplatz 1

10117 Berlin

martin.bruenger@charite.de

\section{Ökonomisierung oder Kommerzialisierung der Gesundheitsversorgung}

\section{Franz Porzsolt}

Die seit den 70er Jahren zunehmende Kommerzialisierung der Gesundheitsversorgung lässt sich zwar nicht umkehren, sollte aber gesteuert werden, um drei Herausforderungen $\mathrm{zu}$ meistern:

- Der Leistungserbringer haftet für gesundheitliche Risiken und Schäden, die als Folge medizinisch unbegründeter Leistungen entstehen. Diese Leistungen lassen sich daran erkennen, dass der durchschnittliche Nutzen irgendeines Akteurs größer ist als der durchschnittliche Nutzen des Patienten.

- Wirtschaftliche Belastungen, die durch unbegründete Gesundheitsleistungen entstehen, können nicht den Versicherten angelastet werden. Deshalb benötigen wir Daten, welche den erzielten Nutzen aus der Sicht verschiedener Akteure unter Alltagbedingungen dokumentieren.

- Die Leistungserbringer sollten selbst auf die Angemessenheit ihrer Leistungen achten, um das eigene Berufsbild nicht zu zerstören. Mit der Umsetzung dieser Forderung kann sofort begonnen werden, weil die Leistungserbringer die zweifellos nützlichen Leistungen kennen.

Diese Ziele können erreicht werden, wenn wir eine gemeinsame Sprache sprechen, gemeinsame Ziele anstreben und gemeinsame Regeln akzeptieren. Beispiele aus drei Ländern zei- gen, dass der Unterschied zwischen Ökonomisierung und Kommerzialisierung $\mathrm{zu}$ beachten ist, um ethische Wertvorstellungen zu schützen und $\mathrm{zu}$ verhindern, dass Versorgungsrisiken kranker Mitbürger aus kommerziellen Gründen billigend in Kauf genommen werden. Diese Behauptungen lassen sich an Beispielen aus drei Ländern belegen.

In den USA hat Atul Gawande 2009 im „New Yorker“ eine lesenswerte Geschichte über die Kostenexplosion im amerikanischen Gesundheitssystem veröffentlicht (Gawande, 2009). Als Ursachen werden ein besserer Service, Malpractice, ,die legale Hölle“", Overutilization und junge, nicht mehr denkende Ärzte diskutiert. Bei einer 